\title{
Binary Phase Diagram of Water/Brij58 Studied by SAXS
}

\author{
S. Fall, B. Pattier, L. Benyayia and A. Gibaud* \\ Laboratoire de Physique de l'Etat Condensé, UMR CNRS 6087, Université du Maine \\ 72085 Le Mans Cedex 09, France
}

\begin{abstract}
The phase diagram of the binary water/Brij58 system has been studied at constant temperature $T=20^{\circ} \mathrm{C}$ first by simple optical observation and then by small angle X-ray scattering. The small angle X-ray scattering patterns in the isotropic diluted phase are analyzed in a quantitative way by refining the experimental measurements with a model assuming hard spherical micelles in interaction. The micelles are described by a poly dispersed core-shell model and the interaction by the Perkus-Yevick hard-sphere model. The fitted parameters are analyzed in detail to determine the way polyoxyethylene blocks are hydrated with respect to the volumic fraction of water. In the more concentrated regime, the crystalline phases that are identified are the $F m 3 m$ cubic, the 2D hexagonal and the lamellar phases. The structural parameters that describe each phases are analyzed and commented. The small angle X-ray scattering study is complemented by the determination of the temperature dependent liquid-solid transition by rheology.
\end{abstract}

PACS: 81.30.-t, 61.05.C-, 64.70.dg

\section{Introduction}

The full understanding of the properties of surfactants when added to oil and water is of considerable interest given their tremendous applications in many scientific fields such as the design of hybrid mesoporous materials, drug delivery, or more prosaically industrial applications of detergents. Among widely used surfactants one finds the non-ionic surfactant of the Brij family characterized by a hydrophilic head made of polyoxyethylene (PEO) and a hydrophobic tail made of an alkyl chain. Quite surprisingly very little information is found in the literature about the phase diagram of the Brij58 (polyoxyethylene(20) cetyl ether). Apart from a neutron study of Brij58 micelles made by Sheffer et al. [1] by small angle neutron scattering and the study done by Mitchell on the phase behavior of polyoxyethylene surfactants with water, no real analysis of the phase diagram is available to the best of our knowledge [1-3].

Our objective in this paper is to present the small angle $\mathrm{X}$-ray scattering (SAXS) determination of the structures developed by the Brij58 when mixed with water from $5 \%$ to $95 \%$ in weight at a constant temperature of $20^{\circ} \mathrm{C}$. The main objective is to present the phase diagram of this binary system and to determine the characteristics of the shell [4]. Brij58 presents a hydrophobic part that is far different from its hydrophilic. It is therefore expected that in SAXS experiments carried out on Brij58, it will be easy to probe the relative importance of the hydrophobic core and of the hydrophilic shell which dictates the scattering properties in the diluted regime of the micellar phase. In particular, one of the key points is to address the way PEO blocks react with water. If one assumes that the density of the PEO block is the one of the melt, a mean value of $1.16 \mathrm{~cm}^{3} \mathrm{~g}^{-1}$ can be accepted. In such a case this translates into an electron density of

* corresponding author; e-mail: Alain.gibaud@univ-lemans.fr the shell of $371 \mathrm{e}^{-} / \mathrm{nm}^{3}$ which is quite different from the one of water which is $334 \mathrm{e}^{-} / \mathrm{nm}^{3}$. The electron density of hydrophobic core of the alkane chain is calculated from the density of hexadecane, for more precision we considered the volumes given by Tanford at ambient temperature $\left(\nu\left(\mathrm{CH}_{2}\right)=0.0269 \mathrm{~nm}^{3}\right.$ and $\left.\nu\left(\mathrm{CH}_{3}\right)=0.0543 \mathrm{~nm}^{3}\right)$ and the partial specific volumes of $-\left(\mathrm{CH}_{2}\right)-$ and $-\left(\mathrm{CH}_{3}\right)$ groups of molten paraffin at $27^{\circ} \mathrm{C}$ given by Luzzati with respectively $2.18 \mathrm{~cm}^{3} \mathrm{~g}^{-1}$ and $1.16 \mathrm{~cm}^{3} \mathrm{~g}^{-1}$, for both of them a value of $281 \mathrm{e}^{-} / \mathrm{nm}^{3}$ is obtained [5,6]. There is therefore a large contrast of electron density between the solvent, the core and the shell in such a system. This large contrast is quite favorable for adjusting the parameters of the model. This means that in addition to determining the phase diagram of Brij58, it is also possible to get a better understanding of hydration of the PEO blocks. We supplement the study of the phase diagram by a study of the binary system water/Brij58 in temperature by rheology, to determine the limit zone of the crystalline phases.

\section{Experimental}

Brij58 of stoichiometric formula $\mathrm{C}_{56} \mathrm{H}_{114} \mathrm{O}_{21}$ is a di-block polymer containing an alkyl hydrophobic tail $\mathrm{C}_{16} \mathrm{H}_{33}$ and a polyoxyethylenic hydrophilic core $-(\mathrm{O}-$ $\left.\mathrm{CH}_{2}-\mathrm{CH}_{2}\right)_{20} \mathrm{OH}$. It was purchased from Aldrich and was used as received. Its specified average molecular weight is $1123.5 \mathrm{~g}$ with a mass density of $0.978 \mathrm{~g} / \mathrm{ml}$ and a melting point between 41 and $49^{\circ} \mathrm{C}$. Brij58 di-block polymers have a critical micelle concentration $(\mathrm{CMC})$ in pure water of about $0.08 \times 10^{-3} \mathrm{~mol} / \mathrm{L}$ at $25^{\circ} \mathrm{C}$. The following experiments are conducted well above the CMC.

SAXS experiments were carried out either in capillaries for the sols or in a teflon cell closed by kapton windows for the gels. The solutions were prepared in sealed vials (water and Brij58) at each composition, aged several weeks and stored at room temperature. To promote the easy dissolution of the surfactant in water, vials were heated at $45^{\circ} \mathrm{C}$ in a "Bain Marie". Sols were then transferred inside borosilicate capillaries of external diameter $1.5 \mathrm{~mm}$. 
The capillaries were sealed with Araldite glue $10 \mathrm{~h}$ prior to doing the experiment in order to avoid any problems during the vacuum evacuation of the chamber.

SAXS measurements were performed at LPEC (Université du Maine, Le Mans) on the Rigaku SAXS spectrometer working with a copper rotating anode operating at $2.2 \mathrm{~kW}$. The incident beam was reflected on a double focusing mirror monochromator and finely collimated through a system of three pinholes so as to produce a beam $200 \mu \mathrm{m} \times 200 \mu \mathrm{m}$ at the sample position. All experiments were carried out at $\lambda=0.154 \mathrm{~nm}$. The scattered intensity was recorded on a Gabriel 2D detector located at $830 \mathrm{~mm}$ from the sample. The transmission through the capillaries was monitored with a pin diode located inside the beam stop. Measurements covered a scattering $q$ range from 0.3 to $2.1 \mathrm{~nm}^{-1}$. A typical acquisition time was about $10000 \mathrm{~s}$. The 2D SAXS patterns were normalized after subtraction of the empty capillary contribution with reference to the scattering of water, i.e. $I=0.0162 \mathrm{~cm}^{-1}$. Intensity was regrouped into $1 \mathrm{D}$ scattering curves using a standard radial averaging procedure. The resulting intensity was therefore obtained in absolute units. Before presenting the results we first shortly recall the theoretical approach used to analyze the data.

Rheology measurements were done at PCI (Université du Maine) on stress-controlled rheometer (AR200, TA instruments) with a cone and plate geometry. The temperature is controlled by a Peltier System. Oscillatory measurements of the storage $\left(G^{\prime}\right)$ and loss modulus $\left(G^{\prime \prime}\right)$ were obtained at $0.1 \mathrm{~Hz}$.

\section{Theoretical approach of SAXS results}

\subsection{Diluted liquid phase}

We have recently discussed the theoretical approach for a diluted phase in which we assumed polydisperse core-shell spherical micelles in interaction [1]. At low concentration but above the CMC the SAXS signal arises from the form factor of the micelles. At higher concentrations, the interaction between micelles that takes place produces a peak arising from the structure factor, $S\left(q, R_{\mathrm{HS}}\right)$ where $q$ is the wave vector transfer and $R_{\mathrm{HS}}$ is the hard sphere interaction radius. There are different models, like the Percus-Yevick (PY), the Rogers-Young (RY) closure with the Ornstein-Zernike integration equation to fit the structure factor for interacting hard spheres. These two models slightly differ from each other in the sense that the PY model is little overestimating the main correlation peak compared to RY [7]. As this difference lies within the instrumental accuracy, the PY approximation was used in this analysis [7]. The full detailed description of the model used in this calculation has been reported in our previous paper with appropriate references to the literature in this field [1]. We simply recall here the expressions of the two alternative models known as the decoupling approximation (DA) and local monodisperse approximation (LMA). In the DA, the scattered intensity in absolute units reads as,

$$
I(q)=r_{\mathrm{e}}^{2} n_{p}\langle P(q, r)\rangle S^{\prime}\left(q R_{\mathrm{HS}}, \eta\right),
$$

where $\eta$ is the volumic fraction of micelles as refined in the PY formalism, $n_{p}=N_{p} / V$ is the number of micelles per unit volume, $N_{p}$ is the total number of micelles, $\langle P(q, R)\rangle$ is the form factor of a micelle averaged over the distribution $f(R)$ of the micellar radius, $S^{\prime}\left(q R_{\mathrm{HS}}, \eta\right)$ is an effective structure factor and $r_{\mathrm{e}}=2.85 \times 10^{-15} \mathrm{~m}$ is the classical radius of the electron. $S^{\prime}\left(q, R_{\mathrm{HS}}\right)$ is related to the structure factor $S\left(q, R_{\mathrm{HS}}\right)$ by the following expression [7]:

$$
S^{\prime}\left(q R_{\mathrm{HS}}, \eta\right)=1+\beta\left(q, R_{\mathrm{HS}}\right)\left[S\left(q R_{\mathrm{HS}}, \eta\right)-1\right],
$$
where $\beta(q, R)=|\langle P(q, R)\rangle|^{2} /\left\langle|P(q, R)|^{2}\right\rangle$.

The DA is valid for small volume fractions and low polydispersity where particles positions are independent of their size. Alternatively one can consider that the positions are fully correlated to the size of the particles (where $\beta(q)=1$ ) in the so-called "local monodisperse approximation" (LMA) and this approximation is more adapted to larger polydispersities even though no LMA or DA works at very large polydispersities. Both approximations are fully described in Ref. [8].

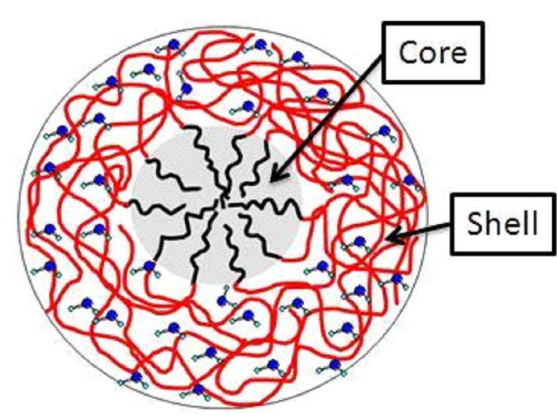

Fig. 1. Illustration of a spherical micelle according to the core-shell model in which the alkane chains are located in the core while the PEO blocks are inside the shell together with bound water molecules.

In the simple core-shell model shown in Fig. 1, in which the core and the shell have a uniform electron density, the form factor yields

$$
\begin{aligned}
& P\left(q, R_{\mathrm{C}}, R_{\mathrm{S}}\right)=\left[4 \pi / 3\left(\rho_{\mathrm{S}}-\rho_{\mathrm{M}}\right)\left(R_{\mathrm{C}}+R_{\mathrm{S}}\right)^{3}\right. \\
& \left.\quad \times F\left(q, R_{\mathrm{C}}+R_{\mathrm{S}}\right)+4 \pi / 3\left(\rho_{\mathrm{C}}-\rho_{\mathrm{S}}\right) R_{\mathrm{C}}^{3} F\left(q, R_{\mathrm{C}}\right)\right]^{2},
\end{aligned}
$$
where

$$
F(q, R)=(\sin q R-q R \cos q R) /(q R)^{3}
$$

is the normalized amplitude scattered by a sphere, $R_{\mathrm{C}}$ and $R_{\mathrm{S}}$ are the radii of the core and the thickness of shell, $\rho_{\mathrm{C}}, \rho_{\mathrm{S}}, \rho_{\mathrm{M}}$ are the electron densities of the core, shell and solvent, respectively.

In the following analysis, we have used the decoupling approximation [8] and local monodisperse approximations $[8,9]$ in which we assumed a polydisperse core and a shell of constant thickness $R_{\mathrm{S}}$. A Gaussian distribution was used to describe the polydispersity of the micelles radii. The Gaussian distribution is given by 


$$
f(R)=\exp \left(-\left(\frac{(R-\langle R\rangle)^{2}}{2 \sigma^{2}}\right)\right),
$$

where, $\langle R\rangle$ is the average radius of the micelles, $\sigma$ is a root mean-square deviation from the mean radius and is related to the polydispersity $\delta$ by $\delta=\sigma /\langle R\rangle$. This model is well described by Pedersen [10] and was used for describing small angle scattering curves for micelles of block copolymers [11-14] and surfactant vesicles [15].

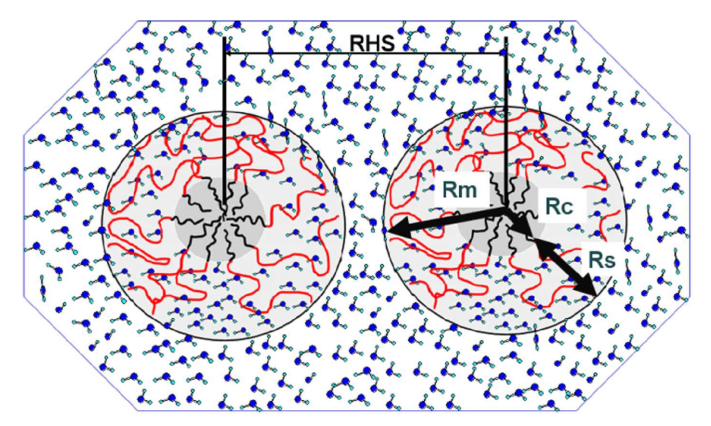

Fig. 2. Illustration of interacting micelles in which the distance $R_{\mathrm{HS}}$ between micelles is presented. $R_{\mathrm{m}}$ is the average radius of the micelle, $R_{\mathrm{C}}$ - the radius of the core and $R_{\mathrm{S}}$ - the thickness of the shell.

Assuming that each micelle has the average volume $\left\langle V_{\text {mic }}\right\rangle$, the volume fraction defined by $\phi=N_{p}\left\langle V_{\text {mic }}\right\rangle / V=$ $n_{p}\left\langle V_{\text {mic }}\right\rangle$, can be inserted in the calculated intensity which yields in the DA and LMA

$$
\begin{aligned}
& I_{\mathrm{DA}}(q)=\frac{r_{\mathrm{e}}^{2} \phi\langle P(q)\rangle S^{\prime}\left(q R_{\mathrm{HS}}, \eta\right)}{\left\langle V_{\mathrm{mic}}\right\rangle}, \\
& I_{\mathrm{LMA}}(q)=r_{\mathrm{e}}^{2} \frac{\phi\langle P(q)\rangle S\left(q R_{\mathrm{HS}}, \eta\right)}{\left\langle V_{\mathrm{mic}}\right\rangle} .
\end{aligned}
$$

For a better understanding of the role of RHS and $P(q)$ please refer to Figs. 2 and 3.

\subsection{Crystalline phases}

For crystalline phases, correlations between the positions of micelles become strong enough to produce Bragg reflections. The width of the Bragg peaks is inversely proportional to the size of the coherent scattering domains. The sequence of the Bragg reflections defined by their Miller indices $h k l$ is used to identify the nature of each phase. The intensity of the Bragg reflections is related to the multiplicity, $m_{h k l}$, of the reflections in the given space group. A Debye-Waller factor can be used to some extent to account for the effect of thermal fluctuations in position of micelles. For monodisperse micelles, the scattering cross-section is proportional to the product of the micellar form factor, $P(q)$ times the structure factor, $m_{h k l}\left|F_{h k l}(q)\right|^{2}$ at a given reflection located at $q=G_{h k l}$ times the exponential Debye-Waller factor,

$$
I(q)=m_{h k l}\langle P(q)\rangle\left|F_{h k l}(q)\right|^{2} \mathrm{e}^{-q^{2} \sigma^{2}} \delta\left(q-G_{h k l}\right),
$$

where $G_{h k l}$ is the modulus of the wave vector transfer corresponding to the location of a particular $h k l$ Bragg reflection.

The calculated scattered intensity is obtained after convolution of the scattering intensity by the instrumen-

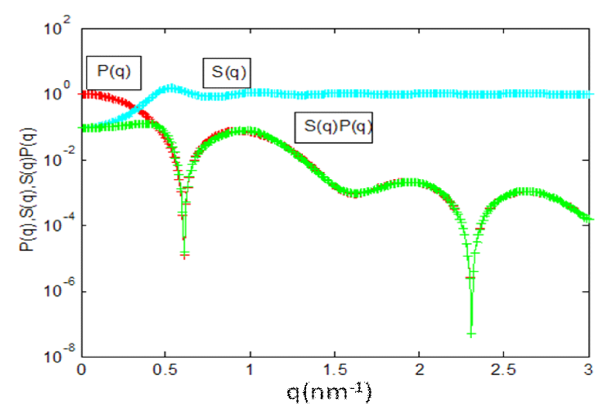

Fig. 3. Illustration of the form factor $P(q)$, the structure factor $S(q)$ and their product $S(q) P(q)$ for non-polydisperse core-shell micelle disregarding the instrumental background showing the effect at low $q$ of the structure factor on the measured intensity.

tal resolution function and by a Lorentzian function with a width equal to $2 \pi / \xi$ related to the coherence length $\xi$ of the scattering domains. This function dominates the broadening of the peaks.

\subsection{Programming}

A MATLAB program (written by Gibaud) has been developed to fit on an absolute scale the observed data to the above description. Eight parameters could be either fixed or adjusted, among which 5 of them describe the form factor, one - the polydispersity of the micelle core radius and 2 of them - the structure factor (depending on the model). These parameters are namely: the radii of the core and the shell $R_{\mathrm{C}}, R_{\mathrm{S}}$, and their corresponding electron densities $\rho_{\mathrm{C}}$ and $\rho_{\mathrm{S}}$, the polydispersity $\delta$, the volume fraction of micelles $\eta$ found in $S\left(q R_{\mathrm{HS}}, \eta\right)$, and the hydrodynamic radius $R_{\mathrm{HS}}$. In the crystalline phases the program can be used to identify the peak positions and the correlation length of the scattering domains.

\subsection{Optical mapping of the phase diagram}

The binary phase diagram was first investigated by optical observations prior to carrying out the SAXS experiments. The results are shown in Fig. 4. At low con-

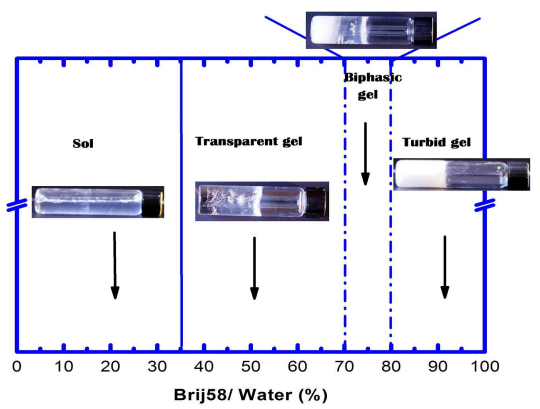

Fig. 4. The domain boundaries of the binary phase diagram of the Brij58 after optical observations. All vials are photographed horizontally so that the liquid phase can be easily recognized by the presence of the horizontal boundary. The full understanding of the phase diagram is now discussed via the analysis of the SAXS data. 
centration the system is liquid until one reaches $35 \% \mathrm{w} / \mathrm{w}$ where it becomes a transparent gel. This gel persists until $70 \% \mathrm{w} / \mathrm{w}$ before one can observe a biphasic system made of a transparent gel in the upper part of the vial and a turbid gel in the lower part. Above $80 \%$ only the turbid gel remains visible. Optical measurements carried out between crossed polarizer and analyzer in the transparent region allowed to evidence the existence of an isotropic gel between $35 \%$ and $50 \%$ and an anisotropic gel between $50 \%$ and $60 \%$. This clearly indicates that a phase transformation occurs around 50\% from an isotropic cubic phase to a phase of lower symmetry which could be likely a 2D hex phase.

\section{SAXS results and discussion}

\subsection{Behavior in the diluted region of the phase diagram $\phi<30 \%$}

The measured and calculated SAXS curves of the binary Brij58/water system are shown in Fig. 5 for six concentrations ranging from 5\%,10\%, 15\%, $20 \%$ and $25 \%$ $\mathrm{w} / \mathrm{w}$.

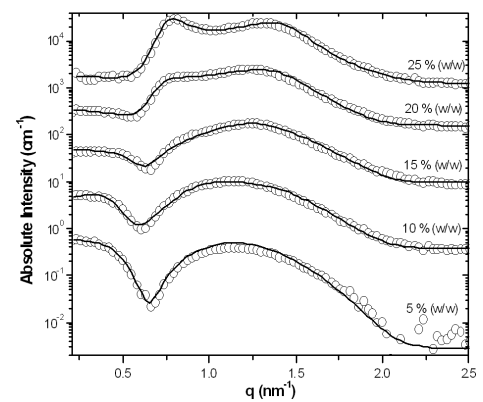

Fig. 5. Measured and calculated SAXS curves presented on an absolute scale at increasing concentration of Brij58 in water. Each curve is offset for clarity by a factor of 10 with respect to the previous one. Sample 5\% $\mathrm{w} / \mathrm{w}$ is presented on an absolute scale.

At $5 \% \mathrm{w} / \mathrm{w}$, the correlation between micelles is very weak as can be seen by the absence of any correlation peak in the low $q$ region of the X-ray pattern. The increasing content of Brij58 produces a lowering of the intensity at small $q$ in full agreement with the development of a strong correlation of the micelles in the solution. At $15 \%$, the location of a correlation peak around $q=0.8 \mathrm{~nm}^{-1}$ becomes quite clear. This peak increases in intensity upon further increase of the Brij58 concentration in the solution. SAXS curves were fitted via the Percus-Yevick hard sphere model in which core-shell spherical micelles interact. The fitting parameters with error bars indicating the range over which no significant changes could be detected in the adjustment are reported in Table I. In the fits, the solvent (i.e. water) and the core electron densities were kept fixed to $334.4 \mathrm{e}^{-} / \mathrm{nm}^{3}$ and $281 \mathrm{e}^{-} / \mathrm{nm}^{3}$, respectively (see column 3 of Table II) while the shell electron density was considered as a free parameter.

As a guideline, the electron densities of pure alkane $\mathrm{C}_{16} \mathrm{H}_{33}$ and pure $\mathrm{PEO}-\left(\mathrm{CH}_{2}-\mathrm{O}-\mathrm{CH}_{2}\right)_{20}-\mathrm{OH}$ blocks in the melt were calculated from the densities of the pure compounds. This yielded $281 \mathrm{e}^{-/ \mathrm{nm}^{3}}$ and $371 \mathrm{e}^{-} / \mathrm{nm}^{3}$, respectively. These values are clearly the maximum values that these two materials may exhibit. In the following calculation, the alkane electron density was fixed at $281 \mathrm{e}^{-} / \mathrm{nm}^{3}$ and all the other parameters were freed. The results presented in Table I yield a core radius ranging from 1.7 to $2 \mathrm{~nm}$. This value is very close to the length, $L=2.02 \mathrm{~nm}$, of the all trans extended chain of alkanes assuming the $\mathrm{C}-\mathrm{C}$ distance to be $0.126 \mathrm{~nm}[16,17]$. The small observed difference means that the alkyl chains in the core slightly overlap. The polydispersity of the core is fairly constant with a value of about $10 \%$ whatever the concentration of the surfactant. The thickness of the shell is slightly decreasing when the concentration of the surfactant increases and is found to be ranging from $2.66 \mathrm{~nm}$ at $5 \%$ to $1.78 \mathrm{~nm}$ at $25 \%$. Correlatively the radius of the core is increasing at higher surfactant concentrations. The thickness of the shell is in fairly good agreement with what has been deduced by Schefer et al. by SANS with values ranging from $2.44 \mathrm{~nm}$ to $2.08 \mathrm{~nm}$ for a two-shell spherical model of Brij58 micelles [1].

TABLE I

Volumic fraction $\Phi$, electronic density of water $\rho_{\text {water }}$, core radius $R_{\mathrm{C}}$, electronic density of the core $\rho_{\mathrm{C}}$, shell radius $R_{\mathrm{S}}$, electronic density of the shell $\rho_{\mathrm{S}}$, polydispersity $\delta$, hard sphere interaction radius $R_{\mathrm{HS}}$, volumic fraction $\eta$ and average radius $R_{\mathrm{m}}$. Columns 2 and 4 correspond to fixed parameters.

\begin{tabular}{|c|c|c|c|c|c|c|c|c|c|}
\hline \multirow[b]{2}{*}{$\begin{array}{c}\Phi_{\text {Brij58/water }} \\
{[\%]}\end{array}$} & \multirow[b]{2}{*}{$\begin{array}{c}\rho_{\text {water }} \\
{\left[\mathrm{e}^{-} / \mathrm{nm}^{3}\right]} \\
\end{array}$} & \multicolumn{5}{|c|}{ Parameters of $F(q)$} & \multicolumn{3}{|c|}{ Parameters of $S(q)$} \\
\hline & & $\begin{array}{c}R_{\mathrm{C}} \\
{[ \pm 0.05 \mathrm{~nm}]}\end{array}$ & $\begin{array}{c}\rho_{\mathrm{C}} \\
{\left[\mathrm{e}^{\left.-/ / \mathrm{nm}^{3}\right]}\right.}\end{array}$ & $\begin{array}{c}R_{\mathrm{S}} \\
{[ \pm 0.01 \mathrm{~nm}]}\end{array}$ & {$\left[ \pm 2 \mathrm{e}^{-} / \mathrm{nm}_{\mathrm{S}}\right]$} & $\begin{array}{c}\delta \\
{[ \pm 0.01]}\end{array}$ & $\begin{array}{c}R_{\mathrm{HS}} \\
{[ \pm 0.1 \mathrm{~nm}]}\end{array}$ & $\begin{array}{c}\eta \\
{[ \pm 0.01]}\end{array}$ & $\begin{array}{c}R_{\mathrm{m}} \\
{[ \pm 0.1 \mathrm{~nm}]}\end{array}$ \\
\hline 5.10 & 334.4 & 1.7 & 281 & 2.66 & 345 & 0.11 & 7.6 & 0.10 & 4.4 \\
\hline 10.20 & 334.4 & 1.9 & 281 & 1.97 & 351 & 0.12 & 6.7 & 0.20 & 3.9 \\
\hline 15.28 & 334.4 & 2.0 & 281 & 1.61 & 353 & 0.12 & 6.6 & 0.27 & 3.7 \\
\hline 20.36 & 334.4 & 2.0 & 281 & 1.89 & 354 & 0.13 & 4.5 & 0.38 & 3.9 \\
\hline
\end{tabular}


Volumic fraction $\Phi$, degree of hydration of the shell $x$, number of water molecules per EO $N_{\text {water/EO, }}$

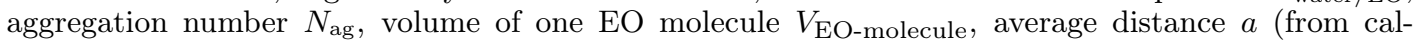
culation), twice the hydrodynamic radius $2 R_{\mathrm{HS}}$ (from fitting), volumic fraction $V_{\text {micelle }} / V_{\mathrm{t}}$ (from calculation) and volumic fraction $\eta$ (from fitting).

\begin{tabular}{c|c|c|c|c|c|c|c|c}
\hline \hline $\begin{array}{c}\Phi \\
{[\%]}\end{array}$ & $\begin{array}{c}x \\
{[\%]}\end{array}$ & $N_{\text {water/EO }}$ & $N_{\text {ag }}$ & $\begin{array}{c}V_{\text {EO-molecule }}\left[\mathrm{nm}^{3}\right] \\
{\left[\begin{array}{c}a \\
{[\mathrm{~nm}]}\end{array}\right.}\end{array}$ & $\begin{array}{c}2 R_{\text {HS }} \\
{[\mathrm{nm}]}\end{array}$ & $V_{\text {micelle }} / V_{\mathrm{t}}$ & $\eta$ \\
\hline 10.20 & 70 & 9 & 45 & 0.109 & 11.9 & 15.2 & 0.21 & 0.10 \\
15.28 & 46 & 3 & 62 & 0.080 & 7.1 & 13.4 & 0.21 & 0.2 \\
20.36 & 46 & 2 & 73 & 0.066 & 9.6 & 9 & 0.24 & 0.27 \\
25.42 & 43 & 2 & 73 & 0.079 & 8.8 & 9 & 0.36 & 0.38 \\
\end{tabular}

The fact that the core radius and the thickness of the shell evolve differently at increasing Brij58 concentration is a clear consequence of the diminution of the water content. When this content is high the shell which likes water expends while the core which dislikes it shrinks.

Among all the fitted parameters the most changing one is the hard sphere radius $R_{\mathrm{HS}}$ of the micelles which reflects the interaction between the micelles. This parameter decreases from $7.6 \mathrm{~nm}$ to $4.3 \mathrm{~nm}$ when the concentration increases from $5 \%$ to $25 \%$. This means that the micelles get closer and closer to each other when the Brij58 concentration increases until they are almost in contact. Indeed at $25 \%, R_{\mathrm{HS}}$ becomes almost equal to the radius of the micelles. It is worth noting that this is in good agreement with the fact that, at 30\%, the micellar phase changes into a crystalline cubic phase. We also observe that the electron density of the shell is less than that of the melt with a typical value close to $355 \mathrm{e}^{-} / \mathrm{nm}^{3}$.

\subsection{Important consequences}

The above fitted parameters can be used to get a better insight of the influence of the increasing concentration of the surfactant both on the role of water solvent on the PEO shell and how the water is distributed in the volume of the solution. The knowledge of the electron density of the shell immediately provides the information on the degree of hydration of the shell $(x)$ according to the following expression:

$$
x=\frac{\rho_{\mathrm{PEO}}-\rho_{\text {shell }}}{\rho_{\text {PEO }}-\rho_{\text {water }}} .
$$

Since the electron density of the shell differs noticeably from the one of pure $\mathrm{PEO}$, it is obvious that an important fraction of water molecules is no more in a pure aqueous phase but is bound to the polar PEO head groups. Here if we assume that $\rho_{\mathrm{PEO}}=371 \mathrm{e} / \mathrm{nm}^{3}$, one can conclude that $x$ is equal to $43 \%$ at $\Phi=15.2 \%$. This means that the shell is abundantly hydrated. The hydration decreases when $\Phi$ increases as intuitively expected.

The volume of water present in the shell can be then calculated at each concentration according to the following expression:

$$
V_{\text {water-in-the-shell }}=x \frac{4}{3} \pi\left[\left(R_{\mathrm{C}}+R_{\mathrm{S}}\right)^{3}-R_{\mathrm{C}}^{3}\right],
$$

which in turn provides the number of water molecules in the shell

$$
N_{\text {water-in-the-shell }}=\frac{V_{\text {water-in-the-shell }}}{V_{\text {one-water-molecule }}} .
$$

This value can be calculated provided the volume of one water molecule is known. It is well admitted that this volume is of the order of $V_{\text {one-water-molecule }}=0.03 \mathrm{~nm}^{3}$. From this information, one can then find the number of water molecules per EO group. Indeed, the number of EO molecules in one micelle is the product of the aggregation number times the number of EO monomers in the Brij58 molecules i.e.

$$
N_{\text {EO }}=20 N_{\text {ag }} \text {. }
$$

The aggregation number can be calculated from the ratio of the total number of electrons in the core to the number of electrons in one alkyl chain, i.e. $Z=129$. It follows that:

$$
N_{\text {ag }}=\frac{4}{3} \pi \frac{R_{\mathrm{C}}^{3} \rho_{\mathrm{C}}}{129},
$$

which yields

$$
N_{\text {water/EO }}=\frac{N_{\text {water-in-the-shell }}}{20 \frac{4}{3} \pi \frac{R_{\mathrm{C}}^{3} \rho_{\mathrm{C}}}{129}} .
$$

This information can be used to further calculate the volume occupied by one EO monomer. Indeed, the volume occupied by the PEO in the shell is the difference between the total volumes of the shell minus the volume occupied by water molecules bound to the EO groups, i.e.

$$
\left\{\begin{array}{l}
V_{\text {PEO-in-the-shell }}=V_{\text {shell }}-V_{\text {water-bond }}, \\
V_{\text {water-bound }}=0.03 N_{\text {water-in-the-shell }}, \\
V_{\mathrm{EO}}=V_{\text {PEO-in-the-shell }} / 20 N_{\text {ag }} .
\end{array}\right.
$$

In addition one can also calculate the number and the volume of free water molecules. For this, we first calculate the ratio of water molecules to the one of Brij58 molecules starting from the definition of weight fraction

$$
\frac{m_{\mathrm{B}}}{m_{\mathrm{B}}+m_{\mathrm{w}}}=\phi=\frac{1}{1+\frac{m_{\mathrm{w}}}{m_{\mathrm{B}}}} \Rightarrow \frac{m_{\mathrm{w}}}{m_{\mathrm{B}}}=\frac{1-\phi}{\phi} .
$$

This translates into a molar ratio 


$$
\frac{n_{\mathrm{w}}}{n_{\mathrm{B}}}=\frac{1-\phi}{\phi} \frac{M_{\mathrm{B}}}{M_{\mathrm{w}}},
$$

where $M_{\mathrm{B}}$ and $M_{\mathrm{w}}$ are the molar masses of Brij58 and water. This ratio is also the ratio of molecules of water per Brij58 molecules. For each micelle there are therefore

$$
N_{\text {water }}=N_{\text {ag }} \frac{1-\phi}{\phi} \frac{M_{\mathrm{B}}}{M_{\mathrm{w}}}
$$

molecules of water. Since part of these molecules is inside the shell it remains a number of free water molecules per micelle equal to

$$
N_{\text {water-free }}=N_{\mathrm{ag}} \frac{1-\phi}{\phi} \frac{M_{\mathrm{B}}}{M_{\mathrm{w}}}-N_{\text {water in the shell }},
$$

which in turn provides the volume occupied by these free molecules of water per micelle. This entitles to calculate the total volume occupied by the micelle plus the one occupied by the free neighbouring water molecules per micelle

$$
V_{\mathrm{t}}=V_{\text {micelle }}+V_{\text {water-free }} \text {. }
$$

As a rule of thumb, we then extract the average distance separating each micelle by taking the cubic root of this volume

$$
a=\left(V_{\mathrm{t}}\right)^{1 / 3} \text {. }
$$

This value can be compared to twice the value of the hydrodynamic radius of the micelle deduced from the fits. In addition the ratio $V_{\text {micelle }} / V_{\mathrm{t}}$ can also be compared to the value of $\eta$ deduced from the fits.

All the parameters resulting from this analysis are tabulated in Table II.

TABLE III

Volume fraction $\Phi_{\text {Brij58/water }}, N_{\text {ag }}$, volume of PEO molecules in the shell $V_{\text {PEO-in-the-shell }}$, number of water molecule in the shell $N_{\text {water-the-shell }}$, number of water molecules per EO group $N_{\text {water/EO, }}$ degree of hydration $x$ (assuming a volume of EO molecule equal to $0.0616 \mathrm{~nm}^{3}$ ), electronic density of the shell $\rho_{\mathrm{s}}$.

\begin{tabular}{|c|c|c|c|c|c|c|c|c|c|c|c|}
\hline $\begin{array}{c}\text { Weight fraction } \\
\phi \quad[\%]\end{array}$ & 30 & 35 & 40 & 45 & 50 & 55 & 60 & 65 & 70 & 85 & 95 \\
\hline $\begin{array}{l}\text { volumic fraction } \\
\Phi_{\text {Brij58/water }}[\%]\end{array}$ & 30.4 & 35.5 & 40.5 & 45.5 & 50.5 & 55.5 & 60.5 & 65.5 & 70.4 & 85.2 & 95.10 \\
\hline phase symmetry & $\begin{array}{l}\text { cubic } \\
F m 3 m\end{array}$ & $\begin{array}{c}\text { cubic } \\
F m 3 m\end{array}$ & $\begin{array}{l}\text { cubic } \\
F m 3 m\end{array}$ & $\begin{array}{l}\text { cubic } \\
F m 3 m\end{array}$ & $\begin{array}{c}\text { cubic } \\
F m 3 m\end{array}$ & $\begin{array}{l}2 \mathrm{D} \text { hex } \\
p 6 m \\
+ \text { cubic }\end{array}$ & $\begin{array}{l}2 \mathrm{D} \text { hex } \\
p 6 m \\
+ \text { cubic }\end{array}$ & $\begin{array}{l}2 \mathrm{D} \text { hex } \\
\quad p 6 m\end{array}$ & $\begin{array}{c}\text { 2D hex } \\
p 6 m \\
+ \text { lamellar }\end{array}$ & $\begin{array}{c}\text { D hex } \\
p 6 m \\
+ \text { lamellar }\end{array}$ & lamellar \\
\hline lattice parameter $a[\mathrm{~nm}]$ & 13.6 & 12.8 & 12.9 & 12.3 & 13 & 7.5 & 7.1 & 7.1 & 17 & 17 & 16.5 \\
\hline $\begin{array}{c}\text { average distance } d[\mathrm{~nm}] \\
\text { between two micelles } \\
\text { neighboring }\end{array}$ & 9.60 & 9.04 & 9.12 & 8.7 & 8.48 & 7.5 & 7.5 & 7.1 & - & - & - \\
\hline
\end{tabular}

\begin{tabular}{c|c|c|c|c|c|c}
\hline \hline $\begin{array}{c}\Phi_{\text {Brij58/water }} \\
{[\%]}\end{array}$ & $N_{\text {ag }}$ & $\begin{array}{c}V_{\text {PEOintheshell }} \\
{\left[\mathrm{nm}^{3}\right]}\end{array}$ & $N_{\text {watertheshell }}$ & $N_{\text {water/EO }}$ & $\begin{array}{c}x \\
{[\%]}\end{array}$ & $\begin{array}{c}\rho_{\mathrm{s}} \\
{\left[ \pm 1 \mathrm{e}^{-} / \mathrm{nm}^{3}\right]}\end{array}$ \\
\hline 5.10 & 45 & 55.4 & 4458 & 5 & 83.5 & 340 \\
10.20 & 62 & 76.4 & 2327 & 2 & 34.7 & 358 \\
15.28 & 73 & 89.9 & 1436 & 1 & 50.3 & 352 \\
20.36 & 73 & 89.9 & 2030 & 1 & 41.8 & 355 \\
25.42 & 73 & 89.9 & 1727 & 1 & 45.8 & 354
\end{tabular}

TABLE IV

Weight fraction $\phi[\%]$, volume fraction $\Phi_{\text {Brij58/water }}[\%]$, phase symmetry, lattice parameter $a$ and the radius of the micelle $R$ as deduced from the assumption that they touch each other in the crystalline phase.

From this table, one can extract general trends concerning the behavior of the PEO blocks and how the micelles evolve when the water amount is changed. First it is clear that the hydration decreases when the surfactant concentration increases going for instance from $x=70 \%$ at a volumic fraction $\Phi=5.1 \%$ to $x=52 \%$ at $\Phi=25.4 \%$. In addition, the aggregation number increases quite a lot from 45 to 73 at these respective volumic fractions. These values are close to the value found in Brij58 solutions by Scheffer et al. $\left(N_{\mathrm{ag}}=71\right)$ by SANS and by Moore et al. $\left(N_{\mathrm{ag}}=49\right)$ [18] by fluorescence emission spectra. The increase of the aggregation number imposes that the number of water molecules per EO decreases as well since more PEO chains and less water are present in the shell. The volume occupied by one EO block is then deduced from this information. It is not surprising that given all the uncertainties we find that this volume changes. The change goes from $0.076 \mathrm{~nm}^{3}$ to $0.1 \mathrm{~nm}^{3}$. Let us note that these values are the values of the volume occupied by an EO block surrounded by water molecules in the micelle. 
The volume of one EO molecule evaluated by Funasaki et al. and Stanislava and Cveto [19, 20] through simulation as $V_{\mathrm{EO}-\text { molecule }}=0.0616 \mathrm{~nm}^{3}$ is fairly different from the one we have found. If we nevertheless assume that this volume is correct, we can back calculate some parameters and compare them to what has been calculated above. We start here with Eq. (15) to determine the number of water molecules per EO assuming that the aggregation number is the right one (this involves the assumption that the radius and the electron density of the core are correct). From this we can derive the volume of the PEO in the shell and thus the volume of water bound to the shell. In turn this gives the number of such molecules, i.e. the degree of hydration, $x$. The calculated values are listed in Table III.

The comparison of the results listed in Tables II and III reveals that the number of water molecules per EO does not differ much between the two assumptions. This is not so surprising because the water volume exceeds the one of the EO in the shell. In this latter assumption where the EO group was inferred to be smaller, we logically find that the degree of hydration is a bit bigger in the latter calculation. In addition, we have back calculated the electron density of the shell as shown in the last column of Table III. We find that it differs from the values which are reported in Table I by less than 6\%. This clearly shows that the influence of the volume of EO is not so important. The reason is due to the fact that the shell contains a lot of water.

Finally from the results shown in Table II we also extract the total volume occupied by one micelle surrounded by water molecules which allows to determine the parameter $a$. This parameter is a characteristic length separating two micelles in solution. As can be seen in Table II, this parameter decreases as expected when the water content decreases. Its value is very similar to the hard sphere radius extracted from the structure factor. This also allows a comparison of the volumic fraction $\eta$ fitted in the structure factor with the one deduced from geometric consideration $\left(V_{\text {micelle }} / V_{\mathrm{t}}\right)$. These two parameters are significantly overestimating the nominal volumic fraction. This could be related to the imperfection of the model used to describe the micelles as hard spheres. Indeed the PEO chains easily mix with water so that the use of an abrupt profile to describe the PEO arms is likely too strong approximation. This statement is enhanced by the fact that the PEO shell is extremely hydrated since most of the volume in the shell is occupied by water. The boundary between the shell and water is thus likely ill defined.

\subsection{Crystalline phases}

Crystalline phases are observed above $30 \% \mathrm{w} / \mathrm{w}$ until $95 \%$ which was the highest concentration we studied. Intense Bragg peaks typical of different symmetries are observed as can be seen in Fig. 6. Below 55\% $\mathrm{w} / \mathrm{w}$, the location of the Bragg peaks is sequenced as $\sqrt{3}, 2, \sqrt{8}, \sqrt{11}, \sqrt{12}, \ldots$ which is typical of a cubic phase having the $F m 3 m$ symmetry.

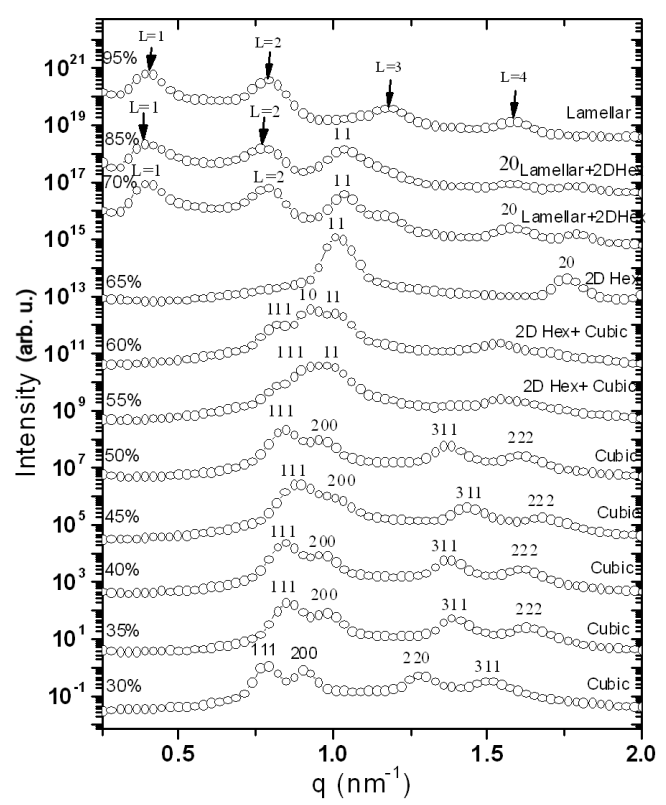

Fig. 6. Typical X-ray scattering patterns for the crystalline phases of the water/Brij58 binary system shown as a function of the increasing fraction of Brij58. All curves are offset for more clarity.

At 55 and $60 \%$ a coexistence of the cubic symmetry with a $2 \mathrm{D}$ hexagonal $P 6 m$ phase is clear. At $65 \%$ the $2 \mathrm{D}$ hexagonal phase is unique with the Bragg peaks located according to the following sequence: $1, \sqrt{3}, 2, \sqrt{7}, 3, \ldots$ It transforms progressively into a pure lamellar phase at $95 \%$ with the Bragg peaks located at multiple values of $q=0.38 \mathrm{~nm}^{-1}$. In the region between 70 and $85 \%$ a mixture of the two phases is observed. These observations fully agree with the ones obtained from the optical determination of the phase diagram reported in Fig. 4. The typical symmetries encountered in the case of the water/Brij58 system are depicted in Fig. 7 and the parameters derived from the Bragg peak positions listed in Table IV.

At $30 \%$ one can see from the peak positions that the cubic phase is typical of the $F m 3 m$ symmetry meaning that the micelles occupy the corner and the middle of the faces of the unit cell. It is quite interesting to extract from the lattice parameter the radius of the micelles assuming they are in contact along the diagonal of the faces and to compare it to the radius of the micelles in the sol. It is straightforward to show that the cubic lattice parameter $a$ is related to the average distance $d$ occupied by a micelle (Fig. 7) and its next neighbour between micelles $d$ by the following relationship:

$$
a \sqrt{2}=2 d .
$$

This yields a distance $d / 2=4.50 \mathrm{~nm}$ at $35 \%$ which is quite close to the average radius $R_{\mathrm{m}}=4.0 \mathrm{~nm}$, obtained in the sol at $x=25 \%$. Let us recall that in this latter calculation $d$ represents the sum of the radius of the micelle plus the water layer surrounding the micelle in the unit 
Cristalline phases of Brij58

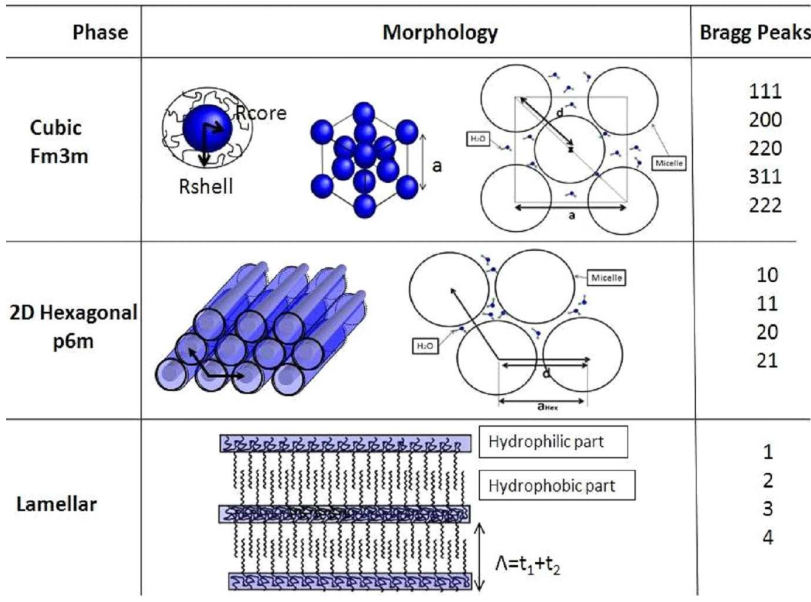

Fig. 7. Morphology of the different crystalline phases of Brij58 together with the indexation of the corresponding Bragg peaks.

cell. The observation of the Fm3m cubic phase shows that the packing of the micelles in the case of Brij58 is optimum as soon as one reaches the weight concentration of $30 \%$ since the fcc structure has the highest compactness. The stability of this phase is quite large since the cubic phase disappears at $\phi=55 \%$. As expected from the diminution of the water content we find that the distance between micelles progressively decreases when the water content decreases. At 55\% weight concentration, the reduction of the water content produces the classical transformation of the spherical micelles into cylindrical ones. This well-known effect is related to the change in the curvature of the micelles the effect of which is characterized by lower curvatures at low water content. This can be translated into a change of the packing parameter,

$$
p=\frac{v}{l A}
$$

(where $v$ is the volume occupied by one molecule of the micelle, $l$ - the length of a molecule and $A$ the area occupied by the head of one molecule) defined by Israelachvili that transforms from $1 / 3$ to $1 / 2$ at this stage [21].

At $55 \%$ to $60 \%$ we have a mixture of $2 \mathrm{D}$ hexagonal $P 6 m$ and a cubic phase $F m 3 m$. In this range of concentration, the lattice parameter $a_{\mathrm{Hex}}$ is twice the average distance occupied by a micelle (Fig. 7),

$$
a_{\mathrm{Hex}}=d .
$$

It is clear that in the $2 \mathrm{D}$ hexagonal phase the lattice parameter $a_{\mathrm{Hex}}$ compared to the one of the cubic $F m 3 m$ phase, decreased consecutively to the diminution of the water quantity. Less free water is available between the micelles and the PEO shell. This translates into a strong diminution of the distance $d$.

Finally at $95 \%$ we observe a true lamellar phase with a lattice parameter of the order of $c=16.5 \mathrm{~nm}$. This means that the length of a single molecule in the lamellar phase is about $8.2 \mathrm{~nm}$. As the carbon-carbon bond length is $1.26 \mathrm{~nm}$ in the alkyl chain $[22,23]$, the alkyl chain $\mathrm{C}_{16} \mathrm{H}_{33}$ length is estimated to be $2 \mathrm{~nm}$ and the hydrophilic part $-\left(\mathrm{O}-\mathrm{CH}_{2}-\mathrm{CH}_{2}\right)_{20} \mathrm{OH}$ to $7 \mathrm{~nm}$.

The calculated lattice parameter for fully extended chains is therefore estimated to be $18 \mathrm{~nm}$. With a measured one equal to $16.5 \mathrm{~nm}$ we can conclude that the hydrophobic and hydrophilic parts slightly overlap in the lamellar phase.

\section{Liquid-solid transition by rheology}

The study of the binary phase diagram water/Brij58 by SAXS at room temperature has been complemented by investigation of a solid-liquid transition. Above a critical temperature $T_{\mathrm{c}}$, the physical gel transforms into a liquid. The determination of the transition temperature (see Fig. 8) is made by monitoring at which temperature the storage modulus reaches $G^{\prime}=10 \mathrm{~Pa}$. As shown in Fig. 8, $T_{\mathrm{c}}$ steeply increases with increasing concentration and becomes almost invariant between $\phi=30$ and 75 weight per cent. $T_{\mathrm{c}}$ decreases sharply when the weight fraction exceeds $75 \%$, mainly due to a lack of water.

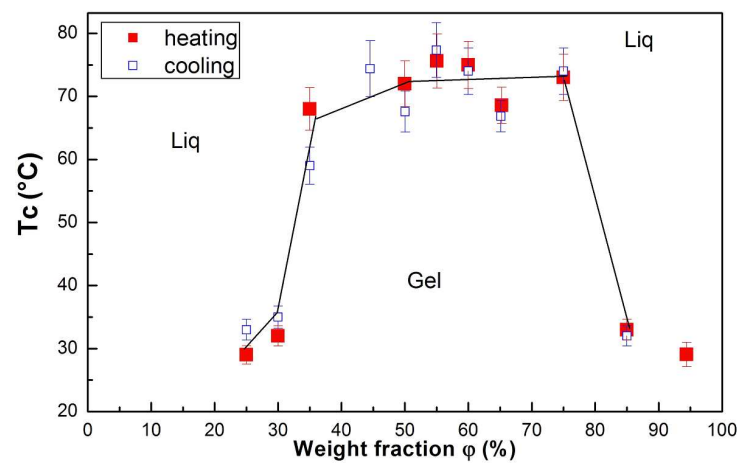

Fig. 8. Solid-liquid state diagram for crystalline phase of the mixture water/Brij58 weight fraction dependence of $T_{\mathrm{c}}$. Solid lines are guides to the eye.

By lowering the temperature, it is possible to observe a reversible transition. Thus, one can say that water/ Brij58 gels are thermo-reversible.

\section{Conclusion}

In this paper the binary phase diagram of Brij58 mixed with water has been investigated by optical observation and SAXS (see Fig. 9). First we identified the boundaries of the different phases by optical observation. Next we used SAXS to determine the exact nature of each phase together with their characteristic parameters. The diluted liquid phase was analysed according to a core shell model of polydisperse spherical micelles. In the concentrated liquid phases, the interaction of the micelles considered as hard polydisperse spheres was investigated in the framework of the Percus-Yevick model. From the fit to the data using a Matlab fitting routine, the parameters describing the morphology of the micelles and their 
interaction were extracted. In particular, the electron density of the shell, the radius of the core, the thickness of the shell, the polydispersity of the core and the hard sphere radius were thus obtained. As a consequence, it was possible to infer the aggregation number and the degree of hydration of the PEO blocks. On the contrary to what we previously published for P123 [4] sentence (P123 stands for the triblock coplymer $\mathrm{EO}_{20}(\mathrm{PO})_{70} \mathrm{EO}_{20}$ where $\mathrm{EO}$ and $\mathrm{PO}$ are the ethylene and propylene oxide blocks), we find that the PEO block is extremely hydrated. We believe that in that case we started with the wrong assumption about the density of the PEO [4].

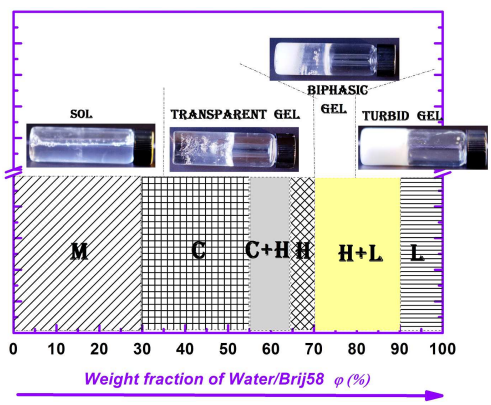

Fig. 9. Room temperature phase diagram of the binary system Brij58/water. M - micellar phase, $\mathrm{C}$ cubic phase, $\mathrm{C}+\mathrm{H}-$ mixture of cubic and $2 \mathrm{D}$ hexagonal phase, $\mathrm{H}-2 \mathrm{D}$ hexagonal phase, $\mathrm{H}+\mathrm{L}-$ mixture of $2 \mathrm{D}$ hexagonal phase and lamellar, $\mathrm{L}$ - lamellar phase.

A specific calculation was then carried out to determine the volume of the water that was not bound to the PEO blocks and a specific distance separating each micelle. This was compared to the hard sphere radius.

We have then identified the crystalline phases. We found a cubic phase with the $F m 3 m$ symmetry between $30 \%$ and $50 \%$, a mixture of a $2 \mathrm{D}$ hex and a cubic phases in the interval 55 to $60 \%$. The $2 \mathrm{D}$ hex phase was clear at $65 \%$ and then a mixture of the $2 \mathrm{D}$ hex and lamellar phases was observed until we reached $85 \%$ where a pure lamellar phase appeared. From the lattice parameters obtained in each phase we extracted the average distance $d$ occupied by a micelle in the cubic and 2D hex phases. One can note that this binary phase diagram of Brij58 (Fig. 9) is quite close to the phase diagram of the $\mathrm{C}_{16} \mathrm{EO}_{12}$ determined by Mitchell by optical microscopy observations, but this latter could not permit to identify the mixed phases hence the use of the SAXS instruments [3]. Finally the temperature domain of existence of gels was monitored by rheology.

\section{Acknowledgments}

The authors are greatly indebted to the region of Pays de la Loire for a grant attribution in the project PERLE to B. Pattier and to the French embassy in Senegal for providing funds for the Ph.D. thesis of S. Fall.

\section{References}

[1] J. Scheffer, R. McDaniel, B.P. Schoenborn, J. Phys. Chem. 92, 729 (1988).

[2] S. Jacques, E. Rocca, M.-J. Stebe, J. Steinmetz, Surf. Coat. Technol. 202, 3878 (2008).

[3] D.J. Mitchell, G.J.T. Tiddy, L. Waring, T. Bostock, M.P. McDonald, J. Chem. Soc., Faraday Trans. 1, 79 (1983).

[4] S.S. Soni, G. Brotons, M. Bellour, T. Narayanan, A. Gibaud, J. Phys. Chem. B 31, 15157 (2006).

[5] F. Reiss-Husson, V. Luzzati, J. Phys. Chem. 68, 3504 (1964).

[6] C. Tandford, J. Phys. Chem. 76, 3020 (1972).

[7] R. Klein, B. D'Aguanno, in: Light Scattering: Principles and Development, Ed. W. Brown, Clarendon Press, Oxford 1996, p. 30.

[8] J.K. Percus, J.K. Yevick, Phys. Rev. 110, 1 (1958).

[9] M. Kotlarchyk, S.H. Chen, J. Chem. Phys. 79, 2461 (1983).

[10] J.S. Pedersen, Adv. Coll. Interface Sci. 70, 171 (1997).

[11] I.W. Hamely, V. Castelletto, Prog. Polym. Sci. 29, 909 (2004).

[12] B. Svensson, U. Olsson, P. Alexandridis, K. Mortensen, Macromolecules 32, 6725 (1999).

[13] I. Goldmints, F.K. Von Gottberg, K.A. Smith, T.A. Hatton, Langmuir 13, 3659 (1997).

[14] I. Goldmints, G.-E. Yu, C. Booth, K.A. Smith, T.A. Hatton, Langmuir 15, 1651 (1999).

[15] M.A. Kiselev, P. Lesieur, A.M. Kisselev, D. Lombardo, M. Killany, S. Lesieur, J. Alloys Comp. 328, 71 (2001).

[16] A. Baptiste, A. Bulou, J.-F. Bardeau, J. Nouet, A. Gibaud, K. Wen, S. Hoeppener, R. Maoz, J. Sagiv, Langmuir 20, 6232 (2004).

[17] C. Tanford, J. Phys. Chem. 78, 2469 (1974).

[18] S.A. Moore, A. Adam, M. Harris, R.M. Palepu, Fluid Phase Equilib. 251, 110 (2007).

[19] N. Funasaki, S. Hada, S. Neya, J. Phys. Chem. 88, 1243 (1984).

[20] K. Stanislava, K. Cveto, Fluid Phase Equilib. 149 233 (1998).

[21] J. Israelachvili, Intermolecular and Surface Forces, 2nd ed., Academic Press, London 1992.

[22] L. Pauling, The Nature of the Chemical Bond, Cornell University Press, New York 1960.

[23] M.J.S. Dewar, H.N. Schmeising, Tetrahedron 11, 96 (1960). 